\title{
Comparison Between Diuretic Urography (IVP) and Diuretic Renography for Diagnosis of Ureteropelvic Junction Obstruction in Children
}

\author{
Mohammad Esmaeili, ${ }^{1}$ Marjan Esmaeili, ${ }^{2, *}$ Fatemeh Ghane, ${ }^{1}$ and Ali Alamdaran ${ }^{3}$ \\ ${ }_{2}^{1}$ Pediatric Nephrology Department, Ghaem Medical Center, Mashhad, IR Iran \\ ${ }_{3}^{2}$ Department of Pediatrics, Iran University of Medical Sciences, Tehran, IR Iran \\ ${ }^{3}$ Department of Radiology, Dr. Sheikh Hospital, Mashhad University of Medical Sciences, Mashhad, IR Iran \\ ${ }^{*}$ Corresponding author: Marjan Esmaeili, Department of Pediatrics, Iran University of Medical Sciences, Tehran, IR Iran. Tel: +98-9153161607, Fax: +98-5117277470, \\ E-mail:esmaeili_82@yahoo.com
}

Received 2014 August 5; Revised 2014 December 22; Accepted 2015 April 9.

\begin{abstract}
Background: Ureteropelvic junction obstruction (UPJO) is one of the most common causes of urinary tract obstruction in children. Several methods are used to diagnose upper urinary tract obstruction including renal ultrasonography(US), intravenous pyelogram(IVP), diuretic renography (DR), magnetic resonance urography (MRU) and antegrade or retrograde pyelography. Nowadays it is suggested to use diuretic renography as the best method for diagnosing of UPJO. There is no comparative study between IVP and DR scan for diagnosis of UPJO in children.

Objectives: The aim of the present study was to compare IVP with furosemide injection and diuretic renography in diagnosis of clinically significant UPJO.

Patients and Methods: This was a cross sectional study performed in 153 UPJO suspected children (121 boys, 32 girls) based on US findings in cases presented with urinary tract infection (UTI), prenatal hydronephrosis, abdominal/flank pain, abdominal mass and hematuria. Renal ultrasound was used as an initial screening tool for detection of urinary tract abnormality. Vesicoureteral reflux (VUR) was ruled out by voiding cystourethrography (VCUG). Serum creatinin, blood urea nitrogen, urinalysis and urine culture was screened in all cases. IVP with furosemide and DR were performed as soon as possible after the mentioned workup.

Results: During a five year period, 46 out of 153 patients were diagnosed as UPJO based on diuretic renography: the age ranged from 4 months to 13 years (mean:3.1 \pm 0.78 years). There was a significant higher (76\%) proportion of UPJO in the boys and in the left side (78\%). The sensitivity of IVP with furosemide injection in diagnosis of UPJO was 91.3\% whereas DR was accepted as standard for diagnostic procedure in diagnosis of UPJO.

Conclusions: Although DR is accepted as the best method for diagnosis of UPJO, we found a small sensitivity difference between IVP and DR in kidneys with normal or near normal function. In many settings such as small cities lacking facilities for advanced isotope imaging technology, use of IVP with diuretic maybe an acceptable procedure for diagnosis of UPJO.
\end{abstract}

Keywords: Ureteropelvic Junction Obstruction, Intravenous Urography, Diuretic Renography, Diruretic Urography, Excretory Urography, Children

\section{Background}

Ureteropelvic junction obstruction (UPJO) is the most common urologic obstructive lesion in childhood which is usually due to intrinsic fibrotic stenosis (1). The incidence of UPJO is approximately 1:1500 children and is one of the most common causes of hydronehrosis detected prenatally by ultrasound. Early diagnosis and management of UPJO is important for marked improvement, determination of prognosis and decision for pyeloplasty. Several methods are used to diagnose UPJO (2). The central ones being ultrasonography (US), intravenous pyelogram (IVP) with furosemide injection or diuretic urography (DU), diuretic renal scan (DRS), retrograde and antegrade pyelography and Whitaker test. Other less commonly used methods, are e.g. MRU, CT and measurement of urinary biochemical markers (3-7). In most urological centers, anatomic and function- al tests are used together, so as not to overdiagnose unobstructed hydronephrosis (pyelocaliectasis, megacalycosis) as UPJL (8), however the choice and the time of radiological imaging is a controversial diagnostic impact.

Since its development in the late 1970s, diuretic renography has been a commonly used test for diagnosing UPJO; in some urological centers, however, excretory urography (IVP) is still used, as it is considered an equally reliable test (9). In addition, isotope imaging facilities are not available everywhere. Although in urologic practice diuretic isotope renal scan is recommended as the most useful procedure for diagnosis of UPJO, there is no documented controlled comparative study between IVP and diuretic renal scan in children (1). A literature review revealed only one prospective study for comparison of IVP and DRS in diagnosing UPJO in adults (10).

Copyright (c) 2016, Growth \& Development Research Center. This is an open-access article distributed under the terms of the Creative Commons Attribution-NonCommercial 4.0 International License (http://creativecommons.org/licenses/by-nc/4.0/) which permits copy and redistribute the material just in noncommercial usages, provided the original work is properly cited. 
Esmaeili M et al.

\section{Objectives}

The aim of the present study was to assess comparative values of two different methods; IVP with furesemide or diuretic urography (DU) and diuretic renal scan or diuretic renography (DR) in diagnosing ureteropelvic junction obstruction in children.

\section{Patients and Methods}

During a 5-year period, between September 2008 and October 2013, all children who referred to our pediatric nephrology clinic with signs and/or symptoms highly suggestive of UPJO such as urinary tract infection, flank/abdominal pain, hematuria and/or previously documented hydronephrosis in prenatal or postnatal ultrasonography enrolled into this prospective study. Informed consent was obtained from the parents. Renal ultrasound was used as an initial screening tool to detect hydronephrosis, urinary stone or any urinary tract abnormalities. Ultrasound was performed by an expert in pediatric sonography. The abdomen and urinary tract were examined in both supine and prone positions. Hydronephrosis was accepted when anteroposterior pelvic diameter (APD) exceeded $10 \mathrm{~mm}$ (11).

In all cases urinalysis and urine culture were set, serum creatinine and urea measured. A renal stone in the uretheropelvic junction was excluded by plain abdominal film and/or urtrasonography. Vesicoureteral reflux (VUR) was ruled out by voiding cystourethrography (VCUG). The patients with UVJO and congenital non obstructive hydroureteronephrosis were excluded. Cases with severe deterioration of renal function and/or gross dilatation of collecting system with a very thin cortex were also excluded to avoid pitfalls in diagnostic judgment. Diuretic renography with TC99-DTPA was accepted as an optimal diagnostic procedure for diagnosis of UPJO.

DU and DR were performed as soon as possible after the treatment of UTI, cystourethrography and renal function assessment (less than 3 months) to confirm obstruction. Diuretic urography was performed in all patients suspected of significant UPJO based on US and DR. In both diagnostic procedures furosemide $(1 \mathrm{mg} / \mathrm{kg}$ ) was administered intravenously 15 - 20 minutes after the injection of radiopaque and radioisotope medium.

DR was carried out in the department for nuclear medicine. All cases were well hydrated. After the completion of a 20 minutes dynamic study, furosemide was injected slowly. The data analysis included differential renal function, half time of tracer clearance after furosemide and renogram curve patterns. In a standardized DTPA diuretic renogram during first 2 to 3 minutes, renal parenchymal uptake was analyzed and compared, allowing computation of differential renal function and depicting functional curves of radioisotope excretion. Results of isotope diuretic renal scan were analyzed qualitatively (visual analysis of scintigrams and renography curves) and quantitatively (time to reach maximum of the kidney cuvre-Tmax washout of the tracer clearance half time) after furosemide injection. A normal renogram curve should show three phases - an initial rapid rise, a peak region and an abrupt decline in activity. A curve showing a plateau-like, gradually increasing or gradually decreasing third phase with little or no change in excretion of dye, so the half-time to tracer clearance $\left(\mathrm{T}_{1 / 2}\right)$ lasting longer than 20 minutes after injection of isotope.

IVP was carried out in good hydration and with bowel preparation. At first a scout film was taken and five minutes after intravenous contrast injection given as bolus another radiograph was made. Successive images were taken after 10 and 20 minutes. Intravenous furosemide $(1 \mathrm{mg} / \mathrm{kg}$ ) was injected slowly over a period of 3 minutes. 20 minutes after the furosemide injection another radiograph was taken. If there was no washout of dye in affected kidney a 60 minute film was made. Further imaging was individualized to the patient until the contrast was eliminated. The urographic signs suggestive of clinically significant obstruction on DU were defined as an ureteropelvic junction that was narrowed, dilated pelvicalyceal system, with delayed excretion of dye on the affected side and no washing out of the dye at post furosemide film.

Comparison of the two procedures and their sensitivity to detect UPJO was statistically analyzed.

\section{Results}

A total of 153 cases (121 boys, 32 girls) were suspected to have UPJO based on US findings. Partially not significant obstruction was confirmed by DR in 107 (69\%). 46 patients (35 boys, 11 girls) had criteria of significant UPJO based on US and DR images. Age ranged from 4 months to 13 years (mean $3.10 \pm 0.78$ years). Presenting signs and symptoms are shown in Table 1.

In 13 patients the right kidney and in 33 cases the left one was affected. DRF of affected kidneys was 25.1 - 48.7\% (mean $41.2 \%$ ) of total renal function. Significant UPJO based on DU was documented in 42 out of 46 patients; sensitivity of DU for diagnosis of UPJO was $91.3 \%$. In four patients a clinically significant UPJO was not confirmed. The range of DRF in these four cases was between $40.5 \%$ and $48.7 \%$ and there were not considerable changes in DRF detected by TC99-DMSA performed 9-16 months later. One out of these 4 cases complained occasionally of mild flank pain after massive fluid intake.

\begin{tabular}{lc}
\hline Table 1. Presenting Signs and Symptoms in 46 Patients With UPJO \\
\hline Signs and Symptoms & Values \\
\hline Abdominal/ flank pain \pm vomiting & $19(41)$ \\
Urinary tract infection & $6(13)$ \\
Prenatal hydronephrosis & $28(60)$ \\
Palpable abdominal mass & $1(2)$ \\
Incidental post natal hydronephrosis & $5(10)$ \\
\hline a Data are presented as No. (\%). & \\
\hline
\end{tabular}




\section{Discussion}

Classic ureteropelvic junction obstruction is defined as congenital hydronephrosis characterized by impaired urine flow from renal pelvis into proximal ureter (12). This is a common renal anomaly in children with an incidence of 1 in 1500, and is the most common cause of hydronephrosis detected prenatally (11). Other clinical features include symptoms of urinary tract infection, abdominal, flank or back pain, palpable renal mass in a newborn or infant and hematuria after minimal trauma $(12,13)$. UPJO usually is secondary to intrinsic fibrotic narrowing of UP junction; in $20 \%$ of cases there is an accessory renal artery supplying the lower pole of the kidney that compresses the ureter.

UPJ obstruction is more commonly (60\%) found on the left side, is more common in males than in females (2:1) and occurs bilaterally in 20 to $40 \%$ of affected children in some reported series (14).

In our study also the left kidney was more commonly involved than the right; male: female ratio was 3:1.

Ultrasonography of urinary system constitutes a cornerstone in the evaluation of renal obstructive disorders. Sonographic examination, however, is operator dependent. The degree of hydronephrosis is used to assist in decision making with regard to management and some prognostic information. However, controversy exists over size cutoffs and significant pathology.

Several methods are used to diagnose upper urinary tract obstruction, the central ones being ultrasonography(US), intravenous pyelogram (IVP) diuretic renal scan and contrast enhanced MRU.

US has many attributes which make it ideal as an initial method for detecting urinary obstruction. It is noninvasive, quick, and portable, requires neither radiographic contrast media nor ionizing radiation and is relatively inexpensive. However, traditional US provides no physoiological or functional data about obstruction. Dehydration, oliguria, and low GFR state of healthy newborns deliver false negative findings. It is also criticized as not being specific for dilatation of renal collecting system. Many conditions rather than obstruction can cause renal dilatation and hence a false-positive result, e.g. extrarenal pelvis, prominent renal vasculature, residual dilatation from previous obstruction, vesicoureteral reflux, congenital megacalycosis, bladder distention and pyelonephritis (15). In addition, hydronephrosis can be found in asymptomatic children with a reported prevalence of $0.19 \%$ in school children by portable ultrasound screening (16). In our study, all children had sonographically moderate to severe hydronephrosis without ureter dilatation. Therefore, to increase the diagnostic accuracy of tools to differentiate between obstructive and non obstructive hydronphrosis, other methods are essential.

Although in a number of urological centers DR is commonly used to diagnose UPJO, in others IVP is used as it is considered an equally reliable test $(9,17)$. Each of the methods has its limitations and each may yield false positive or false negative results. When performing DR special attention should be paid to proper patient preparation, radiopharmaceutical selection, furosemide dosage, and image interpretation to ensure accurate diagnosis.

According to Campbell-Walsh Urology "Excretory urography remains a reasonable first-line option for radiographic diagnosis" of UPJO (18). Urologists using this test to diagnose UPJO report that in most cases IVP allows the anatomic and qualitative functional assessment necessary for the diagnosis and makes it possible to avoid costly imaging studies like a CT angiogram or DR(9).

It seems that diuretic urography in particular is a most valuable test for preoperative and postoperative evaluation of patients with UPJO because the combination of contrast and furosemide creates maximal diuresis, and such "supranormal" conditions are highly conducive to unmasking and documenting UPJ obstruction. Excretory urography makes it possible to evaluate the degree of hydronephrosis; to identify anatomical abnormalities such as a duplicated collecting system or high ureteral insertion; to detect the crossing vessel in many cases (there are usually distinctive radiographic features); and to plan appropriate treatment. However, although IVP helps in the qualitative evaluation of the obstruction, quantitative assessment of it can be difficult (19).

In our study, based on DR findings, diagnosis of significant UPJO could be confirmed in 46 out of 153 hydronephrotic cases. Corresponding results of US and DR were found in $37 \%$ of cases. 46 cases had criteria of significant UPJO in DR and 42 patients in DU.

Historically the IVP has been the classic imaging modality for evaluation of the upper urinary tract. Although with advances in imaging technology the indications for IVP have diminished for a number of clinical situations, it remains an extremely valuable study $(20,21)$, especially in places where no advanced imaging facilities are available, therefore in some centers IVP is substituted for the diuretic renography $(22,23)$.

The role of IVP in the anatomical depiction of renal obstruction such as UPJO is crucial. Collecting system dilatation, with parenchymal changes in the nephrogram and delay in excretion of contrast medium, is characteristic of obstruction (24).

Signs of acute obstruction on IVP are well recognized and include delayed appearance of a persistently dense nephrogram, detrimental changes of parenchyma and delayed excretion of radiocontrast material. Dilatation of the collecting system is less impressive than in chronic obstruction. However, bowel preparation is needed for a better visualization and delayed films, even up to 24 hours, may be required (2). In assessment of the 46 patients in our series the sensitivity of standardized IVP with furosemide injection for UPJO diagnosis was $91.3 \%$. In Tsai's study the sensitivity of IVP for diagnosis of UPJO 
was apparent in only half of patients (25) and in another study DR confirmed UPJO in 35 out of 40 cases that had criteria of significant UPJO in DU (10), which was not in agreement with our study, that maybe due to not using intravenous furosemide in IVP procedure in their study.

IVP has significant limitation in the evaluation of hydronephrosis in patients with a poorly functioning kidney, the newborn period and extremely dilated pelvis (25). Allergic reactions, aggravation of pain and acute renal failure induced by contrast media have been reported (2). In the current study, we observed no complications with IVP.

In nuclear medicine, renal scans are more commonly used than urography as a method of choice to diagnose obstruction in the setting of hydronephrosis. Renography can quantify individual and relative renal function, cortical transit time, and the ante-grade drainage of the collecting systems before and after the administration of furosemide. Technetium-99 m diethylene triamin pentaacetic acid (DTPA) is usually used for detection of upper urinary tract obstruction. A bladder catheter should be inserted, especially if VUR and/or suspected UVJO are present $(12,26)$.

Obstruction at the UPJ is reflected by the half time to washout radioisotope-labeled urine and percentage of tracer retained within the renal pelvis or distal ureter beyond approximately 20 minutes. In the hydronephrotic system, if a quick washout occurs after the furosemide is given, the patient is said to have a dilated, non obstructive system. Prolonged washout times in functioning kidney may support the diagnosis of significant obstruction (27). Evaluating the results of DR, it has been noted that even in technically satisfactory studies, false positive results can result from poor renal function (single kidney glomerular filtration rate $<15 \mathrm{~mL} / \mathrm{min}$ ) and/or hugecapacity systems (28). False negative findings are less common than false positives (10), but they have been observed in both animal experimental models and in clinical practice $(29,30)$. O'Reilly et al noted that such results "can occur in highly compliant (small volume, 'tight') renal pelves, or when there is powerful high-pressure diuresis through partially obstructed systems" (28).

In our study, all of the patients had a significant delay in drainage after injection of furosemide. The sensitivity of DR and DU in UPJO diagnosis was 100\% and 91.3\% in order of frequency.

We hope the study has shown that diagnosing UPJO on the basis of US and DR may result in overdiagnosis; on the other hand, US and DU may give false negative results.

Same as our study, Macleod, compared intravenous pyelography and DTPA scan for assessment of renal function and renal damage, and concluded that the computer processed data in diuretic isotope renal scan gives better results both in the recognition of morphological defects and in the indication and measurement of renal damage (31).

Ebel believed that diuretic urography is more useful during post-operative follow up of UPJO and diuretic renography is useful after 6 months of follow up (32). Lack of availability of surgical findings and post operative fol- low up was limitations to our study.

Also dynamic contrast enhanced MRU appears to provide superior anatomic details compared with renal ultrasound, nuclear scintigraphy and urography, and has no ionizing radiation. However, it is not available at many centers with limited facilities.

Both DR and IVP with furosemide injection are useful for diagnosis of upper urinary tract obstruction such as UPJO. Because of less sensitivity for diagnosis, and complications of IVP such as allergic reactions, aggravation of pain and acute renal failure induced by contrast media and more radiation than DR, DTPA diuretic renal scan for UPJO diagnosis in children is the best alternative method and highly recommended. However in many hospital facilities recent imaging technology is not easily accessible whereas urography is available; therefore, this imaging procedure maybe a useful and appropriate method for diagnosis and following up of upper urinary obstructive lesions in these settings. The authors conclude that excretory urography still remains a valuable test in diagnosing UPJO in patients with near normal renal function or in no deterioration of DRF in the affected kidney over time.

\section{Acknowledgments}

We would like to express our deep gratitude to Mrs. Saeideh Seyed Hoseini, secretary of pediatric department, Dr. Sheikh Hospital for contribution to style and typing of the manuscript.

\section{References}

1. Taha MA, Shokeir AA, Osman HG, Abd el-Aziz Ael A, Farahat SE. Diagnosis of ureteropelvic junction obstruction in children: role of endothelin-1 in voided urine. Urology. 2007;69(3):560-4. doi: 10.1016/j.urology.2006.09.070. [PubMed:17382166]

2. Shokeir AA. The diagnosis of upper urinary tract obstruction. BJU Int. 1999;83(8):893-900. [PubMed:10368226]

3. Muthusami P, Ramesh A. Appearances of the circumcaval ureter on excretory urography and MR urography: A single-center case series. Indian J Radiol Imaging. 2013;23(1):81-5. doi: 10.4103/09713026.113621. [PubMed: 23986621]

4. Kaya C, Caliskan S. Comparison between intravenous urography and computed tomography urography in diagnosing ureteropelvic junction obstruction. Nephrourol Mon. 2012;4(3):585-6. doi:10.5812/numonthly.3402. [PubMed: 23573491]

5. Papachristou F, Pavlaki A, Printza N. Urinary and serum biomarkers in ureteropelvic junction obstruction: a systematic review. Biomarkers. 2014;19(7):531-40. doi: 10.3109/1354750X.2014.943292. [PubMed: 25082300]

6. Taranta-Janusz K, Wasilewska A, Debek W, Waszkiewicz-Stojda M Urinary cytokine profiles in unilateral congenital hydronephrosis. Pediatr Nephrol. 2012;27(11):2107-13. doi: 10.1007/s00467-0122230-9. [PubMed: 22744767]

7. Bartoli F, Penza R, Aceto G, Niglio F, D'Addato O, Pastore V, et al. Urinary epidermal growth factor, monocyte chemotactic protein-1, and beta2-microglobulin in children with ureteropelvic junction obstruction. J Pediatr Surg. 2011;46(3):530-6. doi: 10.1016/j.jpedsurg.2010.07.057. [PubMed: 21376205]

8. Ross JH, Kay R. Ureteropelvic junction obstruction in anomalous kidneys. Urol Clin North Am. 1998;25(2):219-25. [PubMed: 9633577]

9. Yurkanin JP, Fuchs GJ. Laparoscopic dismembered pyeloureteroplasty: a single institution's 3-year experience. J Endourol. 2004;18(8):765-9. doi: 10.1089/end.2004.18.765. [PubMed: 15659899] 
10. Eden CG. Minimally invasive treatment of ureteropelvic junction obstruction: a critical analysis of results. Eur Urol.2007;52(4):9839. doi:10.1016/j.eururo.2007.06.047. [PubMed:17629395]

11. Dhillon HK. Prenatally diagnosed hydronephrosis: the Great Ormond Street experience. Br J Urol. 1998;81 Suppl 2:39-44. [PubMed: 9602794]

12. Chevalier RL, Peter CA. Obstructive Uropathy. In: Anver ED, Harman WE, Niaudet P, Yoshikawa N, editors. Pediatric Nephrology. 6th ed ed. Berlin : Springer; 2009. pp. 1336-75.

13. Homme JL, Foster AA. Recurrent severe abdominal pain in the pediatric patient. J Emerg Med. 2014;46(5):627-31. doi: 10.1016/j. jemermed.2013.11.095. [PubMed: 24582407]

14. Elder JS. Urologic disorders in infants and children. In: Behrman RG, Kliegman RM, Jenson HB, editors. Nelson Textbook of Pediatrics. 17th ed ed. Saunders; 2004. pp. 1783-826.

15. Amis ES, Cronan JJ, Pfister RC, Yoder IC. Ultrasonic inaccuracies in diagnosing renal obstruction. Urology. 1982;19(1):101-5. [PubMed: 7058574]

16. Sheih CP, Liu MB, Hung CS, Yang KH, Chen WY, Lin CY. Renal abnormalities in schoolchildren. Pediatrics. 1989;84(6):1086-90. [PubMed: 2685739]

17. Szydelko T, Kopec R, Kasprzak J, Apoznanski W, Kolodziej A, Zdrojowy $\mathrm{R}$, et al. Antegrade endopyelotomy versus laparoscopic pyeloplasty for primary ureteropelvic junction obstruction. J Laparoendosc Adv Surg Tech A. 2009;19(1):45-51. doi: 10.1089/ lap.2008.0104. [PubMed:18976144]

18. Hsu THS, Streem SB, Nakada SY. Management of upper urinary tract obstruction: Urteropelvic junction obstruction. CampbellWalsh Urology.. Philadelphia: Saunders; 2007;38:1227-53.

19. Elmore J, Kirsch AJ. Assessment of renal obstructive disorders In: Docimo SG, Canning DA, Khoury AE, editors. The Kelalis-KingBelman Textbook of Clinical Pediatric Urology. Informa; 2007. pp. 447-60.

20. Chow JS, Diamond DA. The imaging of reflux and ureteral disease. In: Docimo SG, Canning DA, Khoury AE, editors. The KelalisKing-Belman Textbook of Clinical Pediatric Urology. Informa; 2007. pp. 555-69.

21. Hassan R, Aziz AA, Mohamed SK. Retrocaval ureter: the impor tance of intravenous urography. Malays J Med Sci. 2011;18(4):84-7.

\section{[PubMed: 22589677]}

22. Mathiot A, Liard A, Eurin D, Dacher JN. [Prenatally detected multicystic renal dysplasia and associated anomalies of the genitourinary tract].J Radiol. 2002;83(6 Pt 1):731-5. [PubMed:12149590]

23. Elder JS, Duckett JW. Prenatal urology. In: Gillenwater JY, Grayhack JT, Howards SS, Duckett JW, editors. Adult and Pediatric Uroloy. St. Louis: Mosby; 1991. pp. 1711-810.

24. Platt JF. Urinary obstruction. Radiol Clin North Am. 1996;34(6):111329. [PubMed: 8898787]

25. TsaiJD, Huang FY, Lin CC, Tsai TC, Lee HC, Sheu JC, et al. Intermittent hydronephrosis secondary to ureteropelvic junction obstruction: clinical and imaging features. Pediatrics. 2006;117(1):139-46. doi:10.1542/peds.2005-0583. [PubMed:16396871]

26. Kandeel AA, Elhossainy SA, Elsayed ND. Influence of early (F+0) intravenous furosemide injection on the split renal function using 99mTc-DTPA renography. Nucl Med Commun. 2013;34(4):3548. doi: 10.1097/MNM.ob013e32835e7437. [PubMed: 23376860]

27. Platt JF, Rubin JM, Ellis JH, DiPietro MA. Duplex Doppler US of the kidney: differentiation of obstructive from nonobstructive dilatation. Radiology. 1989;171(2):515-7. doi: 10.1148/radiology.171.2.2649925. [PubMed: 2649925]

28. O'Reilly P, Aurell M, Britton K, Kletter K, Rosenthal L, Testa T. Consensus on diuresis renography for investigating the dilated upper urinary tract. Radionuclides in Nephrourology Group. Consensus Committee on Diuresis Renography. J Nucl Med. 1996;37(11):1872-6. [PubMed: 8917195]

29. Koff SA, Thrall JH. Diagnosis of obstruction in experimental hydroureteronephrosis. Urology. 1981;17(6):570-7. doi:10.1016/00904295(81)90079-0. [PubMed: 7245448]

30. McCarthy CS, Sarkar SD, Izquierdo G, Dunn EK, Strashun AN, Herskowitz M, et al. Pitfalls and limitations of diuretic renography. Abdom Imaging. 1994;19(1):78-81. [PubMed: 8161913]

31. Macleod MA, Houston AS. A comparison of three methods of assessing renal function. Eur J Nucl Med. 1981;6(5):183-9. [PubMed: 7018910]

32. Ebel KD, Bliesener JA, Gharib M. Imaging of uretero-pelvic junction obstruction with stimulated diuresis. With consideration of the reliability of ultrasonography. Pediatr Radiol. 1988;18(1):54-6. [PubMed:3277139] 